1. Bremer JPet al. Diabetes Care 2009, 32 1513-7.

2. Freckmann $G$ et al. Diabetes Technol The 2010, 12, 221-31.

\title{
Selbst Blutzuckermessen Neue Geräte sind besonders präzise
}

- Die „nächste Generation“ von Blutzuckermessgeräten, zeichnet sich neben einfacher Bedienbarkeit durch eine besonders hohe Messgenauigkeit und Zuverlässigkeit aus.

„Basis jeder Insulintherapie ist die regelmäßige Blutzuckerselbstmessung." Daran erinnerte Prof. Dirk Müller-Wieland aus Hamburg. So würden etwa Hypoglykämien von älteren Typ-2-Diabetikern oft nicht wahrgenommen, wie eine Laborstudie ergeben hat [1]. Prof. Thomas Koschinsky, München, Diabetologe im Ruhestand und Experte für Point-of-CareMessgeräte, betonte: „Selbst wenn der Patient alles richtig macht, können fehlerhaft oder ungenau arbeitende Geräte seine Bemühungen konterkarieren.“ Denn: „Längst nicht alle 100 Messgeräte mit CEZertifizierung erfüllen die geltende ISO 15197:2003, obwohl diese eine Abweichung von 20\% nach oben und unten zulässt." So wurden in einer Studie 27 CE-zertifizierte Blutzuckermessgeräte geprüft, aber nur 16 von ihnen wurden den Vorgaben gerecht [2].

Kein Problem mit der geltenden und der demnächst erwarteten neuen ISO-Norm mit nur noch je $15 \%$ erlaubter Abweichung haben die Contour ${ }^{\circledR}$
Blutzuckermessgeräte des Unternehmens Bayer. „Das neue Messgerät mit den Conotur ${ }^{\oplus}$-NEXT-Sensoren bleibt sogar innerhalb eines Zehn-Prozent-Bereichs nach oben und unten“, so Bern Harrison, Wissenschaftler am Bayer-Standort Mishawaka, USA. Möglich werde dies durch das Multi-Puls-Prinzip mit sieben separaten Messimpulsen innerhalb der Messzeit und durch eine innovative Sensortechnologie: „Ihre Grundlage ist eine enzymatische Reaktion mit Glukosedehydrogenase-Flavin-Adenin-Denukleotid und einem eigens entwickelten Phenothiazin-Mediator. Dies verhindert Messfehler etwa durch Paracetamol, Ascorbinsäure und andere häufige Störsubstanzen sowie durch hohe Hämatokritwerte“, so Harrison. „Für den Benutzer stehen zwei Bedienlevel zur Verfügung: das Ablesen des Blutzuckerspiegels in drei einfachen Schritten - ohne Codierung - oder auch die Nutzung zusätzlicher Funktionen wie das Abrufen von Durchschnitts- und die Vorgabe von Zielwerten.“

Quelle: Pressegespräch bei der Diabetes-Messe am 2.3.2012 in Münster, Veranstalter: Bayer HealthCare

\section{Typ-2-Diabetes Inkretinbasierte Therapie über Blutzuckersenkung hinaus effektiv}

- So wirksam wie Sulfonylharnstoffe (SH), aber deutlich besser verträglich - das zeichnet inkretinbasierte Therapien aus. Auch gibt es Hinweise auf protektive Wirkungen am Herzen und an den Betazellen.

„Inkretinbasierte Therapien haben einen festen Stellenwert in der Therapie bei Typ-2-Diabetes", sagte Prof. Juris J. Meier aus Bochum. DPP-4-Hemmer und GLP-1-Analoga senken den Blutzucker vermittelt über eine glukoseabhängige Steigerung der Insulinsekretion und Reduktion des Glukagonspiegels. Sie sind ähnlich effektiv wie SH [1]: Beim Vergleich von Sitagliptin (z.B. Januvia ${ }^{\circledR}$ mit Glipizid, jeweils kombiniert mit Metformin, war die $\mathrm{HbA}_{1 \mathrm{c}}$-Senkung identisch. Der Effekt war umso größer, je höher der Ausgangswert war. „Auch bei Werten von über $10 \%$ kann der $\mathrm{HbA}_{1 \mathrm{c}}$ um mehr als 3\% reduziert werden“, erklärte Meier die hohe Effektivität der Therapie auch bei primär schlechter Stoffwechseleinstellung.

Laut Meier besteht die Attraktivität inkretinbasierter Therapien in der guten Verträglichkeit und Sicherheit: Hypoglykämien, mit SH häufig und mit einem bis zu vierfach erhöhten kardiovaskulären Risiko assoziiert, waren in der Studie von Nauck mit Sitagliptin, das nun auch für Patienten mit moderater bis terminaler Niereninsuffizienz zugelassen und in reduzierter Dosis (50 und $25 \mathrm{mg}$ Tabl.) verfügbar ist, deutlich seltener (5\% vs. 32\%). Beide Substanzklassen der inkretinbasierten Therapie erhöhen nicht das Körpergewicht. Besonders Sitagliptin zeichnet sich durch gute gastrointestinale Verträglichkeit aus.

Zudem gibt es Hinweise auf Schutzeffekte vor progredientem Betazellverlust und kardiovaskuläre Ereignisse. Eine Betazellregeneration, wie tierexperimentell gezeigt, konnte zwar nicht bestätigt werden. Bunck et al. wiesen aber nach, dass die Funktion der Betazellen über drei Jahre stabil gehalten werden konnte [2]. SH erhöhen die Gesamt- und die kardiovaskuläre Sterblichkeit, besonders bei Patienten mit Myokardinfarkt in der Anamnese, wie eine dänische Registerstudie mit mehr als 100000 Patienten bei mittlerem Follow-up von 3,3 Jahren ergab [3]. Für inkretinbasierte Therapien dagegen ließen Analysen mit kleinen Fallzahlen einen Trend zur Reduktion kardiovaskulärer Ereignisse erkennen, so Meier. Weitere Antworten werden laufende prospektive kardiovaskuläre Endpunktstudien mit DPP-4-Hemmern und GLP-1-Analoga in 2-3 Jahren liefern. koc

Quellen: Symposium am 26.11.2011 in Hamburg, Veranstalter: MSD Sharp \& Dohme, Herstellerinformationen 